\title{
Effects of Post-Activation Potentiation on Athletic Performance: A Mini-Review
}

\author{
Daniel Jaffe*, Jennifer Hewit and Timothy Costantini \\ Department of Physical Education, United States Military Academy, USA \\ *Corresponding author: Daniel Jaffe, Department of Physical Education, United States Military Academy, West Point, New York, USA
}

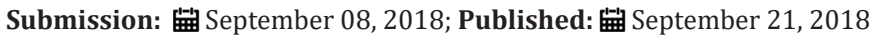

\begin{abstract}
Background and Purpose: Post-Activation Potentiation (PAP) is an increase in the contractile ability of a muscle that is demonstrated subsequent to a maximal or near-maximal bout or exertion $[1,2]$. The purpose of this investigation was to examine the effects that PAP has on athletic performance.

Method: The review included articles from peer-reviewed journals with sufficient data related to the purpose and focus of the study. Inclusion criteria include randomized control trials, systematic reviews and meta-analysis published since 1993. Key words included: "post-activation potentiation", "complex training", "power" and "athletic performance."
\end{abstract}

Results: Twenty relevant studies were identified; various experimental protocols were employed, including both acute and chronic effects of postactivation potentiation on physical performance. All studies were published 1993 through 2015, providing a robust overview of experimentation over the last 25 years.

Discussion and Conclusion: Among studies analyzed in this mini-review, the consensus reached regarding the effects of post-activation potentiation on athletic performance was relatively positive. Most studies followed similar data collection strategies, with slight variations in load, rest-interval, and modality of assessment. The majority of these studies demonstrated significant increases in athletic performance in concert with moderate duration recovery intervals. More research is necessary to identify optimal load patterns and recovery intervals for promoting enhanced athletic performance.

\section{Introduction}

There is great interest in the functional significance of post activation potentiation (PAP). If applied correctly, techniques to induce PAP could enhance performance in high-intensity, short duration sports such as track sprinting events, jumping events, and even to a limited degree in sports such as Football. Defined as the increase in force of a plyometric exercise immediately following a similar heavy resistance exercise, PAP is normally applied using complex training, which includes performing a high-load weight training exercise before executing a plyometric exercise with similar biomechanical demands [2]. Heavy resistance exercise is normally low velocity, while movements such as vertical jumping involve high velocity $[3,4]$. However, more traditional methods separating the weight training and plyometric sessions could also induce PAP. It remains to be seen which method is more effective at eliciting a performance benefit, and how PAP could be best exploited to enhance sport performance.

Chatzopoulos et al. [2] recruited 15 amateur team game (basketball, handball, volleyball, and soccer) players with at least 5 years of experience in their respective sport. All subjects regularly performed resistance training 2 to 3 times a week before the study. During the first session of the study, participants tested 1 repetition maximum (1RM) back squat and were familiarized with the equipment and experimental conditions. For the first testing session, participants executed 10 single sets at 90 percent of $1 \mathrm{RM}$ for the back squat. After 3 minutes of rest, the subjects performed 3 trials of a 10-meter sprint, with the best time recorded. For the second testing session, the same procedure was used but with a 5-minute rest between the squat and sprint. No significant enhancement of running performance was noted after execution of the back squat during the 3-minute rest trial was noted ( $p>0.05)$. The researchers hypothesized that during the first 3 minutes after the heavy stimulus, fatigue is more dominant in the muscle than PAP. The results of the 5-minute rest trial showed an improvement in running time and running acceleration $(\mathrm{p}<0.05)$. The 5 -minute rest likely provided enough time for fatigue to become less of a factor that PAP [2].

McCann and Flanagan [3] recruited 8 male and 8 female NCAA Division I volleyball athletes with at least 1 year of experience with resistance and plyometric training. Vertical jumps were performed on a force plate embedded in the floor, and jump height was col- 
lected using a Vertec jump apparatus. Subjects completed 5 repetitions of back squats at 80 percent $5 \mathrm{RM}$, followed by two sets of five vertical jumps with 4 minutes rest between the squats and vertical jumps. Subjects were then given 15 minutes rest before repeating the same testing procedure but with 5 minutes between exercises. Subjects performed the same procedure 48 hours later, but with 5 repetitions of hang cleans at 50 percent 5RM. Results showed that there was no significant difference in between the squat and hang clean tests. In both, the 4-minute rest interval showed significant improvement in vertical jump performance from the baseline vertical jump data $(\mathrm{p}=0.016)$, while the 5-minute rest interval showed no significant improvement on baseline vertical jump ( $p=0.117)$. The study also concluded that vertical jump height and peak ground reaction force were not affected by gender [3].

High levels of muscular power and sprinting performance are essential in many activities required of team sport athletes $[5,6]$. PAP requires an athlete to perform a heavy resistance exercise followed by a biomechanically similar plyometric exercise such as a sprint or a vertical jump [7-11]. Optimal conditions to maximize PAP are till currently in question, with multiple studies have tested varying exercises and rest intervals in an effort to optimally induce PAP [12]. Wong et al. [9] conducted a study using sled towing as the PAP stimulus and found mixed results among subjects regarding PAP in a 30-meter sprint. The researchers concluded that PAP was highly individualized based on rest time, with some subjects showing evidence of PAP at differing rest intervals.

Bevan et al. [7] recruited 16 professional rugby players. At the time of entry, all players completed a power phase that incorporated Olympic lifts and complex training. Participants tested 3RM squat in a familiarization session 48 hours before the testing session. Subjects completed baseline 10-meter sprints in the beginning of the testing session after a warm up routine. Subjects performed a 3RM squat as the preload stimulus, then performed a 10-meter sprint after 4, 8, 12, and 16 minutes of rest. No significant increase in sprint performance among subjects for any of the rest intervals was noted $(\mathrm{p}=0.401)$. However, the study reported large variance of responses between individuals based on rest time. Approximately 53.3 percent of subjects performed their best sprint time 8 minutes after the squat preload stimulus. While results were not significant for the whole group, an adequate individualized rest time could show improvement in sprint time due to PAP [7].

Linear sprinting speed and acceleration are extremely important in sports performance. Sled towing is a method often used to improve sprint performance and lower body power and strength. Sled towing could also lead to acute outcomes such as Postactivation Potentiation (PAP). The first mechanism of PAP is thought to be phosphorylation of myosin regulatory light chains, causing an influx of calcium to create more active binding sites. The second mechanism is activation of higher order motor units, which allows type II fibers to produce higher force and velocity [10]. Vandenboom et al. [11] found that by inducing phosphorylation of myosin light chains in mouse extensor digitorum with a $5 \mathrm{~Hz} 20$ second conditioning stimulation, myosin light chain phosphate content was increased nine-fold, and peak isometric force was increased by as much as $15 \%$ [11].

While PAP is an interesting phenomenon, its applicability to sport is still in question. With the present knowledge of PAP, it would require relatively significant amounts of equipment and time to induce PAP prior to sporting events and have the potentiated state last longer than only a few minutes in the beginning of the event. Future studies should explore the decay rate of PAP, to determine exactly how long a muscle remains in a potentiated state following a contraction. It would also be beneficial to determine the effects of repeatedly inducing PAP using maximal contractions. This would mean exploring fatigue as a result of multiple maximal contractions and comparing it to PAP to see if the extent of muscle fatigue changes in relation to the amount of PAP experienced as a result of repeated contraction. The current studies focus solely on PAP as an acute response and exploring if there are any chronic adaptations would increase the current knowledge of PAP, and possibly reveal a way to better implement PAP into sport.

\section{References}

1. McBride JM, Nimphius S, Erickson TM (2005) The acute effects of heavyload squats and loaded countermovement jumps on sprint performance. J Strength Cond Res 19(4): 893-897.

2. Chatzopoulos DE, Michailidis CJ, Giannakos AK, Alexiou KC, Patikas DA, (2007) Postactivation potentiation effects after heavy resistance exercise on running speed. J Strength Cond Res 21(4): 1278-1281.

3. McCann MR, Flanagan SP (2010) The effects of exercise selection and rest interval on postactivation potentiation of vertical jump performance. J Strength Cond Res 24(5): 1285-1291.

4. Hodgson M, Docherty D, Robbins D (2005) Postactivation potentiation: Underlying physiology and implications for motor performance. Sports Med 35(7): 585-595.

5. Chiu LZF, Fry AC, Weiss LW, Schilling BK, Brown LE, et al. (2003) Postactivation potentiation responses in athletic and recreationally trained individuals. J Strength Cond Res 17(4): 671-677.

6. Docherty D, Hodgson MJ (2007) The application of postactivation potentiation to elite sport. International Journal of Sports Physiology and Performance 2(4): 439-444.

7. Bevan HR, Cunningham DJ, Tooley EP, Owen NJ, Cook CJ, et al. (2010) Influence of postactivation potentiation on sprinting performance in professional rugby players. Journal of Strength \& Conditioning Research 24(3): 701-705.

8. Sale DG (2002) Postactivation potentiation: Role in human performance. Exercise \& Sport Sciences Reviews 30(3): 138-143.

9. Wong MA, Dobbs IJ, Watkins CM, Barillas SR (2017) Sled towing acutely decreases acceleration sprint time. Journal of Strength \& Conditioning Research. 31(11): 3046-3051.

10. Rassier D, Macintosh BR (2000) Coexistence of potentiation and fatigue in skeletal muscle. Brazilian Journal of Medical and Biological Research 33(5): 499-508.

11. Vandenboom R, Grange RW, Houston ME (1993) Threshold for force potentiation associated with skeletal myosin phosphorylation. American Journal of Physiology 265(6 Pt 1): C1456-C1462.

12. Kilduff LP, Owen N, Bevan H, Bennett M, Kingsley MIC, et al. (2008) Influence of recovery time on postactivation potentiation in professional rugby players. Journal of Sports Sciences 26(8): 795-802. 
(c) (i) Creative Commons Attribution 4.0 International License

For possible submissions Click Here

Submit Article

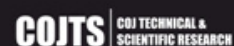
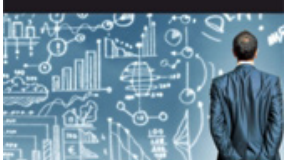

- Freely accessible online immediately upon publication

- Authors retain the copyright to their work

- Licensing it under a Creative Commons license

- Visibility through different online platforms 